\title{
Experimental demonstration of the sensory basis of the size-weight illusion
}

\author{
SERGIO CESARE MASIN \\ University of Udine, Udine, Italy \\ and \\ LOREDANA CRESTONI \\ University of Padua, Padua, Italy
}

\begin{abstract}
An important part of the literature on the size-weight illusion ascribes great importance to expectancies as determinants of the illusion itself. The aim of the experiments reported here was to establish whether the size-weight illusion was sensorial or was caused directly by an expectancy. In Experiment 3, which was the crucial experiment, subjects first looked at an object (from a $3 \times 5$, size $\times$ weight, factorial design), and then the object was hidden from view. Immediately after the object was hidden from view, the subjects lifted the object and rated its heaviness. It was expected that any possible motor set, or any perceptual or cognitive expectancy, should persist during the lifting. The size-weight illusion did not occur in Experiment 3. Therefore, it was concluded that the illusion was of sensory origin.
\end{abstract}

The size-weight illusion may occur when two objects of different size but equal weight are compared. Usually, the smaller of the two objects is felt as being heavier than the larger one. The illusion is clearly due to an influence of size on heaviness. This influence shows up also when the heaviness of single objects is rated. Small objects are usually rated heavier than larger ones of equal weight.

Anderson (1970) proposed the following informationintegration model of the size-weight illusion. Actual weight $(W)$ and the actual size $(S)$ would give rise to the subjective scale values, $w$ and $s$, respectively. The scale value $w$ would be directly related to $W$; that is, $w$ would be the scale value when the lifted object is not seen. Also, a scale value of $w^{*}$ would be generated in direct relation to (the potential $W$ corresponding to) the heaviness expected by the subject on the basis of the size of the object seen. When the lifted object is seen, its heaviness would be given by the following average:

$$
\eta=x w^{*}+(1-x) w,
$$

where $x$ is a weight coefficient varying in the interval $[0,1)$. If $w$, or $w^{*}$, is generated alone, $w$ gives rise to felt heaviness, but $w^{*}$ does not.

Anderson $(1970,1972)$ obtained results that confirmed Model 1 and at the same time disproved any possible ratio model, such as Sjöberg's (1969). Birnbaum and Veit (1974) obtained results that also disproved ratio models, but at the same time showed that Model 1 did not give perfect predictions of the illusion. They concluded that

We thank N. H. Anderson for helpful comments on the first draft of the manuscript. Mail may be directed to $S$. C. Masin, Institute of Philosophy, Pedagogics, and Teaching of Modem Languages, Via Antonini 8,33100 Udine, Italy. the moderate violations of Model 1 indicated that other factors besides expectancy might play a role in the sizeweight illusion.

Birnbaum and Veit (1973; Birnbaum, Kobernick, \& Veit, 1974) proposed that subjective scale values due to an expectancy depend upon the subjective correlation between perceived cue and judged variables. In the case of the size-weight illusion, the cue variable is size and the judged variable is heaviness. Thus, they proposed that

$$
w^{*}=r_{\sigma, \eta} \sigma,
$$

where $r_{\sigma, \eta}$ is the subjective correlation between seen size $(\sigma)$ and felt heaviness $(\eta)$. Clearly, the value of $r_{\sigma, \eta}$ depends on the past experience of the subject. It follows that $w^{*}$ would be generated after the activation of some memory compartment in which $r_{\sigma, \eta}$ is stored.

Figure 1 shows how Birnbaum and Veit's theory of expectancy could be incorporated in a flow-chart version of Model 1. The stimuli $S$ and $W$ give rise to the internal representations $s$ and $w$, respectively. The perceived size, $\sigma$, of the object is assumed to be generated from $s .{ }^{1}$ Since $\sigma$ is used to recompute the correlation $r_{\sigma, \eta}$, it must enter a memory compartment where that recomputation is made. The circled numbers indicate two possibilities. It may be that $w^{*}$ is generated (1) directly from $s$ (for example, due to $S-S$ conditioning), or (2) from the memory compartment where $r_{\sigma, \eta}$ is stored. If only Possibility 1 were true, it would mean that the size-weight illusion was sensorial in origin. If only Possibility 2 were true, it would mean that the illusion was produced directly by an expectancy.

Thus, an important part of current literature (see, also, Brunswik \& Herma, 1951; Davis \& Roberts, 1976; Müller \& Schumann, 1889; Nakatani, 1985; Uznadze, 


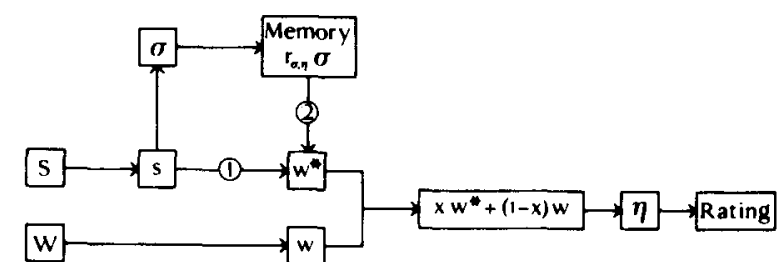

Figure 1. Flow-chart version of the information-integration model, Model 1, of the size-weight illusion. (See text for explanation.)

1966) lays stress on the role of expectancy, sometimes giving the impression that an expectancy is the main, or even the sole, determinant of the size-weight illusion. It seems important, therefore, to test Possibilities 1 or 2 to determine which is true. The aim of the experiments reported below was to obtain such a test.

Experiments 1 and 2 were control experiments. In Experiment 1 , subjects lifted weights without being able to see the objects lifted (no size-weight illusion). In Experiment 2 , subjects lifted a weight while seeing it (this is the situation in which the the size-weight illusion has always been reported to occur).

Experiments 3 and 4 were test experiments. In Experiment 3, an object was shown to the subject and then hidden from view; while still hidden, the object was then lifted by the subject. If only Possibility 1 was true, the size-weight illusion would not occur during the time that the object was being lifted, because the $w^{*}$ that was generated by $s$ before the lifting would now have vanished, since it was not kept in memory. If only Possibility 2 was true, seeing the object before lifting it would activate the memory compartment (that is, activate an expectancy) that generates $w^{*}$, thus producing the size-weight illusion while the object is being lifted without being seen. (In point of fact, the occurrence of the illusion in Experiment 3 would preclude a decision about whether only Possibility 2 was true or whether both Possibilities 1 and 2 were true.)

In Experiment 4, the subject lifted the hidden object before seeing it. After the object had been lifted, it was exposed and the subject rated its heaviness. When an object is seen after it has been lifted, the felt $\eta$ is memorized and only $w^{*}$ is now being generated. The internal representation, $w^{*}$, cannot, however, be integrated with $w$, because $w$ has now vanished. Besides, $w^{*}$ cannot give rise to any felt heaviness. Thus, in Experiment 4 the subject could rate only the $\eta$ that was memorized before he/she had seen the object, and consequently no sizeweight illusion was expected to show up.

\section{METHOD}

\section{Subjects}

The subjects were 120 university students who were asked to participate in the experiment as they entered the General Psychology Department at the University of Padua.

\section{Stimuli}

The stimuli were gray (reflectance .45 ) cylindrical plastic bottles. To vary their weight, they were filled with lead shot and cot ton. A string was tied to the top of each bottle. The free end o the string was then tied to a ring to be used for lifting. The ring weighed $2 \mathrm{~g}$ and was $4 \mathrm{~cm}$ in diameter. Fifteen bottles were con structed according to a $3 \times 5$ (size $\times$ weight) factorial design. Th. size of a bottle was $4.5 \times 7,7.5 \times 13$, or $9 \times 17 \mathrm{~cm}$ in width anc height, respectively. The five levels of weight were 150, 225, 340 510 , and $765 \mathrm{~g}$. Two standards were used-one a $4.5 \times 7 \mathrm{~cm}$ bottle weighing $100 \mathrm{~g}$, and the other a $9 \times 17 \mathrm{~cm}$ bottle weighing $1,150 \mathrm{~g}$.

\section{Apparatus}

A $220 \times 100 \mathrm{~cm}$ (width $\times$ height) plywood screen, mounted oi top of a 75-cm-high table, was used to hide the weights. As soon as a subject entered the experimental room, he/she was asked tc stand $40 \mathrm{~cm}$ from the front of the screen as determined by a mark on the table. The experimenter, who was on the other side of the screen, then placed the string of a bottle into two pulleys, one or the experimenter's side of the screen and one on the subject's side with the ring used for lifting on the subject's side. The lengths o the strings varied such that the height of the ring was always $160 \mathrm{~cm}$ The bottles were hefted by pulling down the ring.

A sliding panel with a rectangular $22 \times 45 \mathrm{~cm}$ opening was in serted in the screen. The opening served to display the bottles tc subjects. When the sliding panel was pulled completely across tc the left of the subject, the bottle was visible. When the panel was pulled all the way to the right, the bottles could not be seen.

Above the rectangular opening was a rectangular $42 \times 26 \mathrm{~cm}$ win dow through which the subject could see the string and the ex perimenter. Allowing subjects to see the experimenter assured therr that the experimenter was not manipulating the weights when the bottles were hidden.

\section{Procedure}

The subject was told to insert his/her right index finger in the ring and to pull down with a regular and smooth vertical move ment. The ring was at the same height as the top edge of the win dow through which the subject communicated with the experimenter The distance of movement was about $20 \mathrm{~cm}$ from the top edge o the window to the bottom edge. The screen was never touched $b$ : the subject.

The two standards were presented first. In each experiment, hal of the subjects were first presented with the light standard and the with the heavy standard; the order was reversed for the other hal of the subjects. The subjects were told that these light and heav standards should be called 1 and 20 , respectively. All the othe weights that followed had to be rated using numbers in proportior to those assigned to the standards. The heavier the object, the large the number assigned. The 15 bottles were then presented in a ran dom order that differed for each subject. When that task was com pleted, the two standards were re-presented. Then the 15 bottle were re-presented, in a new random order. The remaining part o the procedure differed according to experiment:

Experiment 1 (20 subjects). The subject lifted the bottles withou seeing any of them.

Experiment 2 (20 subjects). The subject was asked to look at th bottle through a rectangular opening, while he/she was lifting th bottle.

Experiment 3 (40 subjects). The subject was asked to look at th bottle. After 1-2 sec, the experimenter moved the sliding panel al the way to the subject's right, thus hiding the bottle. The subjec was told to lift the bottle immediately after it was hidden. 

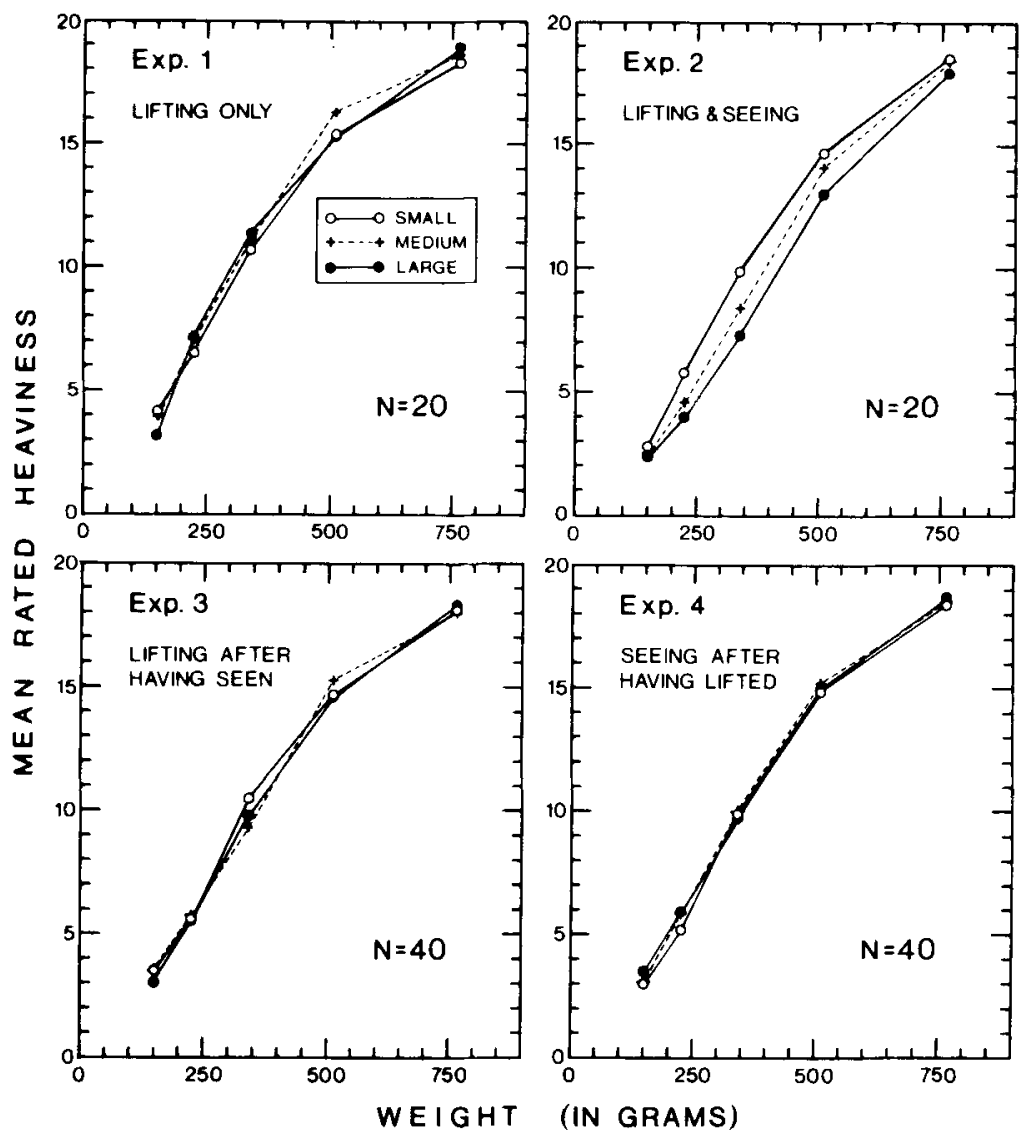

Figure 2. Illustration of the results of Experiments 1-4. The size-weight illusion occurred only in Experiment 2. (See text for explanation.)

Experiment 4 (40 subjects). The subject lifted the bottle without seeing it. As soon as the lifting procedure ended, the experimenter moved the sliding panel all the way to the subject's left, thus exposing the bottle. The subject was told to rate the heaviness only after the bottle became visible.

In a given experiment, the standards were presented with the same procedure used for the other weights in that experiment.

\section{RESULTS AND DISCUSSION}

The results are depicted in Figure 2. The mean rated heaviness is represented on the ordinates; bottle weights, in grams, are represented on the abscissas. The open dots, pluses, and filled dots refer to small, medium, and large bottles, respectively. An analysis of variance (using arithmetic means of the two subjects' ratings) showed the effect of size to be statistically significant in Experiment 2 $[F(2,38)=11.3, p<.0002]$ but nonsignificant in Experiments $1[F(2,38)=.96], 3[F(2,78)=.68]$, and 4 $[F(2,78)=1.39]$. The predictions of the results from $\mathrm{Ex}$ periments 1,2 , and 4 (that is, that the size-weight illusion had to occur only in Experiment 2) are therefore confirmed by the statistical analyses. The result from Experiment 3 confirms Possibility 1, that is, that the sizeweight illusion has a sensory basis.

The interaction was statistically significant in Experiment $3[F(8,312)=2.3, p<.05]$ but nonsignificant in
Experiments $1[F(8,152)=1.33], 2[F(8,152)=1.36]$, and $4[F(8,312)=.66]$. Thus, the graphs resulting from Experiment 3 slightly diverge from perfect parallelism, suggesting that memory exerted some slight influence on rated heaviness. However, that influence was not sufficient to produce the size-weight illusion. It may be that the moderate violations of Model 1 reported by Birnbaum and Veit (1974) could be attributed to a memory effect.

The pattern of data in Experiment 2 does not fit a ratio model at all, since a ratio model would have yielded a linear fan shape.

It seems reasonable that, when a subject lifts an object 1-2 sec after the object has been hidden, a motor set (Davis \& Roberts, 1976; Müller \& Schumann, 1889) or a cognitive or perceptual expectancy (Bimbaum, 1975; Nakatani, 1985; Uznadze, 1966) still persist during lifting. Thus, Experiment 3 invalidates motor-set and expectancy theories of the size-weight illusion, insofar as motor-set and expectancy are conceived as direct determinants of an internal representation $\left(w^{*}\right)$ to be integrated with an internal representation (w) of actual weight.

It has been shown experimentally that in the case of the size-numerosity illusion (Birnbaum \& Veit, 1973; Birnbaum et al., 1974), or the "distance-from-fulcrum"weight illlusion (Birnbaum, 1975), a manipulation of the correlation between apparent size and numerousness, or 
between apparent distance and heaviness, affects the sign and magnitude of the corresponding illusion. Given these findings, it seems very plausible that the size-weight illusion, too, may be affected by a manipulation of $r_{a, n}$ (Figure 1), which would seem to suggest that the sizeweight illusion may be affected by expectancy. Although, according to Birnbaum and Veit (1973), the expectancy is incorporated in $w^{*}$ (Equation 2), the results of Experiment 3 show that expectancy may influence heaviness only after $w^{*}$ has been generated. This implies that expectancy could affect heaviness (1) through an influence on $w^{*}$, after $w^{*}$ has been generated and only when $w^{*}$ and $w$ are simultaneous, or (2) through a change in the weight coefficient $x$ in the information-integration model, Model 1-that is, a change in the relative importance of the two internal representations, $w$ and $w^{*}$.

\section{REFERENCES}

ANDERSON, N. H. (1970). Averaging model applied to the size-weight illusion. Perception \& Psychophysics, 8, 1-4.

ANDERSON, N. H. (1972). Cross-task validation of functional measurement. Perception \& Psychophysics, 12, 389-395.

Birnbaum, M. H. (1975). Expectancy and judgment. In F. Restle, R. M. Shiffrin, N. J. Castellan, H. R. Lindman, \& D. B. Pisoni (Eds.), Cognitive theory (Vol. 1, pp. 107-118). Hillsdale, NJ: Erlbaum.

Birnbaum, M. H., Kobernick, M., \& Vit, C. T. (1974). Subjective correlation and the size-numerosity illusion. Journal of Experimental Psychology, 102, 537-539.

Birnbaum, M. H., \& VerT, C. T. (1973). Judgmental illusion produced by contrast with expectancy. Perception \& Psychophysics, 13, 149-152.

Birnbaum, M. H., \& VerT, C. T. (1974). Scale-free tests of an additive model for the size-weight illusion. Perception \& Psychophysics, 16, 276-282.

Brunswix, E., * Herma, H. (1951). Probability learning of perceptual cues in the establishment of a weight illusion. Journal of Experimental Psychology, 41, 281-290.

DAvis, C. M., RoberTs, W. (1976). Lifting movements in the sizeweight illusion. Perception \& Psychophysics, 20, 33-36.

Müller, G. E., SchumanN, F. (1889). Über die psychologischen Grundlagen der Vergleichung gehobener Gewichte. Archiv für die Gesamte Physiologie, 45, 37-112.

Nakatani, K. (1985). Application of the method of fixed set to the size-weight illusion. Psychological Research, 47, 223-233.

SJöвERG, L. (1969). Sensation scales in the size-weight illusion. Scandinavian Journal of Psychology, 10, 109-112.

Uznadze, D. (1966). The psychology of set. New York: Consultants Bureau.

\section{NOTE}

1. The relationship between $s$ and $\sigma$ is unknown. No attempt at specifying such a relationship is made here, because the reasoning that follows does not depend on whether or not $s$ and $\sigma$ coincide.

(Manuscript received August 24, 1987; revision accepted for publication March 2, 1988.) 